\title{
Diffusion of Conserved Charges in Relativistic Heavy Ion Collisions ${ }^{\dagger}$
}

\author{
Jan A. Fotakis ${ }^{1, *}$, Moritz Greif ${ }^{1}$, Gabriel S. Denicol ${ }^{2}$ and Carsten Greiner ${ }^{1}$ \\ 1 Institut für Theoretische Physik, Johann Wolfgang Goethe-Universität, Max-von-Laue-Str. 1, \\ D-60438 Frankfurt am Main, Germany; greif@th.physik.uni-frankfurt.de (M.G.); \\ carsten.greiner@th.physik.uni-frankfurt.de (C.G.) \\ 2 Instituto de Física, Universidade Federal Fluminense, UFF, Niterói, RJ 24210-346, Brazil; gsdenicol@id.uff.br \\ * Correspondence: fotakis@th.physik.uni-frankfurt.de \\ + Presented at Hot Quarks 2018 - Workshop for Young Scientists on the Physics of Ultrarelativistic \\ Nucleus-Nucleus Collisions, Texel, The Netherlands, 7-14 September 2018.
}

Published: 17 April 2019

check for updates

\begin{abstract}
We discuss the diffusion currents occurring in a dilute system and show that the charge currents do not only depend on gradients in the corresponding charge density, but also on the other conserved charges in the system-the diffusion currents are therefore coupled. Gradients in one charge thus generate dissipative currents in a different charge. In this approach, we model the Navier-Stokes term of the generated currents to consist of a diffusion coefficient matrix, in which the diagonal entries are the usual diffusion coefficients and the off-diagonal entries correspond to the coupling of different diffusion currents. We evaluate the complete diffusion matrix for a specific hadron gas and for a simplified quark-gluon gas, including baryon, electric and strangeness charge. Our findings are that the off-diagonal entries can range within the same magnitude as the diagonal ones.
\end{abstract}

Keywords: Diffusion; Multiple Charge Conservation; Heavy Ion Collisions; Transport Theory

\section{Introduction}

To study the properties of dense and hot nuclear matter, much attention was given to the ultrarelativistic collisions of heavy ions. In such collisions, a new state of nuclear matter is produced, where the quarks and gluons are no longer confined in hadrons, but form a strongly coupled plasma - the quark-gluon-plasma (QGP). Especially, one is interested in the thermal properties of this new state, such as the transition from the hadronic phase to the deconfined phase. Therefore, much effort was invested into uncovering the phase diagram of nuclear matter. To explore the phase diagram in high baryon density collider experiments, such as the Relativistic Heavy Ion Collidier's (RHIC) beam energy scan (BES) program [1-3], collisions are performed at lower collision energies. To understand the evolution of such collisions, it is essential to understand the transport properties of nuclear matter. For example, much attention has been focused on the electric conductivity of quark matter [4-12]. However, since the net-charge density of nuclear matter produced in high energy heavy ion collisions is almost homogeneously vanishing and dissipative effects due to diffusion are hard to observe [13], diffusion processes received less attention. On the other hand, this picture changes at lower energies since the baryo chemical potential can reach high values compared to the temperature $[14,15]$. Thus, strong gradients in chemical potentials of the conserved charges are expected. It is therefore essential to study the effects of these gradients in order to better understand the evolution of heavy ion collisions at lower energies.

In first order relativistic fluid dynamics, the net charge diffusion current $j_{q}^{\mu}$ of the conserved charge $q$ is determined by the Navier-Stokes term $j_{q}^{\mu}=\kappa_{q} \nabla^{\mu} \alpha_{q}$, where $\kappa_{q}$ is the so-called diffusion 
coefficient and the diffusion current is driven by the spatial gradient $\left(\nabla^{\mu} \equiv \Delta^{\mu v} \partial_{v}\right)$ in the thermal potential $\alpha_{q} \equiv \mu_{q} / T$, with $\mu_{q}$ the charge chemical potential and $T$ the temperature. Here, we also introduced the projector $\Delta^{\mu v} \equiv g^{\mu v}-u^{\mu} u^{v}$, where $g^{\mu v}$ is the metric and $u^{\mu}$ the fluid velocity. In heavy ion collisions we must assume at least three conserved charges: baryon number $(B)$, strangeness $(S)$ and electric charge $(Q)$. In general, hadrons and quarks are carriers of multiple charge types. This implies a direct coupling between the diffusion currents of different charges of the system. For example, a hadronic system can consist of strange baryons and thus a gradient in baryo chemical potential not only generates a diffusive flow of baryon charge but also diffusion currents in strangeness. We therefore generalize the Navier-Stokes term by including this explicit coupling as

$$
\left(\begin{array}{c}
j_{B}^{\mu} \\
j_{Q}^{\mu} \\
j_{S}^{\mu}
\end{array}\right)=\left(\begin{array}{ccc}
\kappa_{B B} & \kappa_{B Q} & \kappa_{B S} \\
\kappa_{Q B} & \kappa_{Q Q} & \kappa_{Q S} \\
\kappa_{S B} & \kappa_{S Q} & \kappa_{S S}
\end{array}\right) \cdot\left(\begin{array}{c}
\nabla^{\mu} \alpha_{B} \\
\nabla^{\mu} \alpha_{Q} \\
\nabla^{\mu} \alpha_{S}
\end{array}\right),
$$

where the diffusion coefficient is now replaced by a coefficient matrix with entries $\kappa_{q q^{\prime}}$. Therefore, to describe the diffusive evolution during a heavy ion collision, the diffusion matrix must be evaluated. The diagonal entries of this matrix are the usual diffusion coefficients of the corresponding charge. The off-diagonal entries explicitly introduce the coupling discussed above, and to the best of our knowledge, have never been evaluated before.

We present the derivation of the generalized expression for the diffusion current shown in Equation (1) from kinetic theory and calculate the complete charge diffusion matrix for the first time, for the three charges listed above. We perform this task for a dilute hadron resonance gas (HRG) and for a kinetic theory toy model of the QGP with conserved baryonic, strangeness and electric charge by solving the (linearized) Boltzmann equation $k_{i}^{\mu} \partial_{\mu} f_{\mathbf{k}}^{i}=\sum_{j=1}^{N_{\text {sp. }}} C_{i j}\left(x^{\mu}, k^{\mu}\right)$, with the methods presented in [11]. In this calculation scheme, the single-particle distribution of each particle species is expanded in a Chapman-Enskog series, i.e., in a gradient expansion [16,17], $f_{\mathbf{k}}^{i} \sim f_{0 \mathbf{k}}^{i}+\epsilon f_{1 \mathbf{k}}^{i}+\epsilon^{2} f_{2 \mathbf{k}}^{i}+\cdots$, where $f_{j \mathbf{k}}^{i}$ is the $j$-th order solution of the expansion and $\epsilon$ is a book-keeping parameter which counts the order of thermal gradients. In contrast to the previous work in [11], we disregard any external field. To yield the Navier-Stokes coefficients, the first order corrections to the local-equilibrium single-particle distribution function are evaluated and diffusion currents are calculated using kinetic theory. The diffusion coefficients are then the result of the comparison of these currents and Equation (1). Further details on these calculations can be found in [18] and will also be presented in a forthcoming more detailed publication [19]. We use natural units, $\hbar=c=k_{B}=1$, and we denote the Minkowski metric as $g^{\mu \nu}=(1,-1,-1,-1)$.

\section{Results and Discussion}

In order to provide some understanding on the magnitude of the off-diagonal coefficients compared to the usual diagonal terms, we need to carry out the calculation scheme for a specific system with specified differential cross sections describing all possible particle interactions. For the first evaluation, we restrict ourselves to the simplified case of elastic, isotropic, binary scatterings but, in the case of hadronic matter, employ $\sqrt{s}$-dependent cross sections. We use available experimental data [20] and for all unknown interactions we employ constant cross sections and orientate ourselves on Refs. [21-23] with values between $3-35 \mathrm{mb}$. Apart from applying the calculation scheme to a hadronic system consisting of the 19 most lightest hadrons $\left(p, \bar{p}, n, \bar{n}, \pi^{0}, \pi^{ \pm}, K^{0}, \bar{K}^{0}, K^{ \pm}, \Lambda^{0}, \bar{\Lambda}^{0}, \Sigma^{0}, \Sigma^{ \pm}, \bar{\Sigma}^{0}, \bar{\Sigma}^{ \pm}\right)$, we also assume a simplified model for a conformal QGP, consisting of three flavors of massless quarks and gluons. For this system, we fix the shear viscosity to entropy density ratio to $\eta / s=1 /(4 \pi)$ such that the total cross section runs with temperature as $\sigma_{\text {tot }}=0.72 / T^{2}[24,25]$.

We show our results in Figure 1 for the coefficient matrix in Equation (1). The results for the hadronic system are plotted on a temperature interval ranging from $50 \mathrm{MeV}$ to $160 \mathrm{MeV}$, and the results for the simplified QGP are shown from $160 \mathrm{MeV}$ up to $250 \mathrm{MeV}$. The baryo chemical potential 
is set to the values $\mu_{B}=0,300,600 \mathrm{MeV}$ and to mimic a heavy ion collision, the chemical potentials $\mu_{Q}$ and $\mu_{S}$ are adjusted such that we recover exact isospin symmetry and vanishing net strangeness. First, in our evaluation we find that the diffusion matrix is symmetric, $\kappa_{q q^{\prime}}=\kappa_{q^{\prime} q}$, which is supported by Onsager's theorem $[26,27]$ and therefore the problem is reduced to evaluating six coefficients.

We note that the results for the hadronic system show a much richer dependence in temperature and baryo chemical potential than the dependencies in the conformal QGP model. This is due to the vast number of different scales introduced by the massive hadronic species. In contrast, in the conformal system the only scale is temperature because the baryo chemical potential also obeys $\mu_{B} \sim T$. In this case, evaluating the unitless diffusion coefficients leads to a (almost) constant value, $\kappa_{q q^{\prime}} / T^{2}=$ const. The strong dependencies on the baryo chemical potential solely emerge in the baryo sector of the diffusion coefficients, $\kappa_{B q^{\prime}}$, because only corresponding charge carriers can contribute to the diffusion coefficient. For example, in a system without any particles with strangeness, all coefficients in the strangeness sector vanish, $\kappa_{S q^{\prime}}=0$.

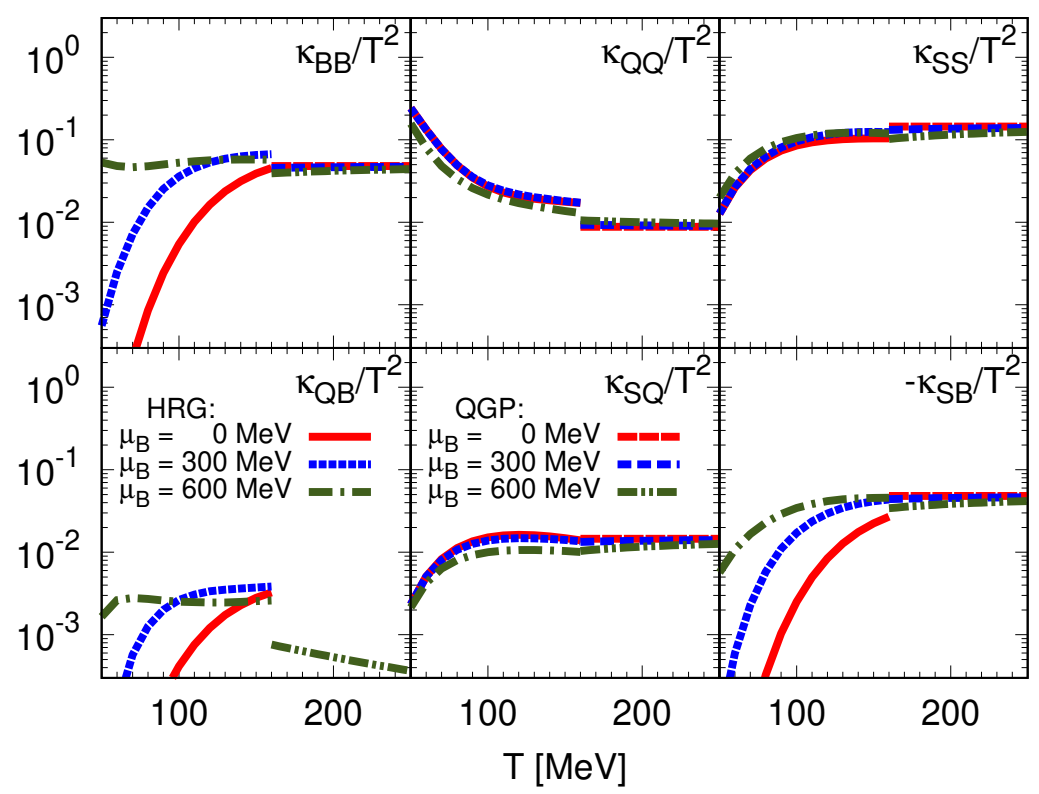

Figure 1. Evaluated diffusion coefficients $\kappa_{q q^{\prime}} / T^{2}$ of a system with 19 hadronic species plotted for temperatures below $160 \mathrm{MeV}$ and for a conformal system of massless quarks and gluons calculated for temperatures larger than $160 \mathrm{MeV}$.

\section{Conclusions}

In order to describe diffusion processes in heavy ion collisions, we suggest that the complete diffusion coefficient matrix, and therefore the implied cross-coupling of diffusion currents, must be taken into account. We presented the first results for the coefficient matrix of two systems consisting of baryon, strangeness and electric charge: for a hot hadron gas and a simplified model for a QGP using kinetic theory. The calculated transport coefficient can be used in fluid dynamic simulations or other models of heavy ion collisions. We suspect that these models could play a crucial role in understanding processes that take place in low energy and high density heavy ion collisions at RHIC BES, NICA or FAIR.

The off-diagonal entries of the coefficient matrix describe the coupling between the diffusive flows of different charges, and our findings show that these can reach similar magnitudes as the diagonal terms. We therefore suggest that the coupling of diffusion currents should not be neglected in simulations of high density collisions, as we suspect that it will lead to significant effects in the evolution of the charge currents. For example, one can imagine that due to the magnitude of $\kappa_{S B}$, gradients in baryo chemical potential could lead to the separation of net strangeness during the 
evolution of a high density collision, which would lead to measurable effects e.g., in the rapidity distribution of strange particles. However, the scope of these effects depends on the dynamic evolution of the thermal potentials $\alpha_{q}$. Therefore, our future investigations will focus on this direction. Moreover, the calculated diffusion coefficients should be accessible from (hadronic and partonic) transport models and a comparison to such evaluations would be desirable.

Funding: The authors M.G., J.A.F. and C.G. acknowledge support from the Deutsche Forschungsgemeinschaft (DFG) through the grant CRC-TR 211 "Strong-interaction matter under extreme conditions". M.G. acknowledges the support from the "Helmholtz Graduate School for Heavy Ion research". J.A.F. acknowledges support of the "Stiftung Polytechnische Gesellschaft", Frankfurt am Main. Furthermore, G.S.D. would like to thank Conselho Nacional de Desenvolvimento Científico e Tecnológico (CNPq) for financial support.

Acknowledgments: The authors thank Harri Niemi for fruitful discussion. This work was supported by the Helmholtz International Center for FAIR within the framework of the LOEWE program launched by the State of Hesse.

Conflicts of Interest: The authors declare no conflict of interest. The founding sponsors had no role in the design of the study; in the collection, analyses, or interpretation of data; in the writing of the manuscript, nor in the decision to publish the results.

\section{References}

1. Aggarwal, M.M.; Ahammed, Z.; Alakhverdyants, A.V.; Alekseev, I.; Anderson, B.D.; Arkhipkin, D.; Averichev, G.S.; Balewski, J.; Barnby L.S.; Baumgart, S.; et al. (STAR Collaboration). An Experimental Exploration of the QCD Phase Diagram: The Search for the Critical Point and the Onset of De-confinement. 2010. [arXiv:1007.2613].

2. Mohanty, B. for STAR Collaboration. STAR experiment results from the beam energy scan program at RHIC. J. Phys. 2011, G38, 124023. [arXiv:1106.5902].

3. Mitchell, J.T. for PHENIX Collaboration. The RHIC Beam Energy Scan Program: Results from the PHENIX Experiment. Nucl. Phys. 2013, A904-A905, 903c-906c. [arXiv:1211.6139].

4. Aarts, G.; Allton, C.; Amato, A.; Giudice P.; Hands, S.; Skullerud, J.-I. Electrical conductivity and charge diffusion in thermal QCD from the lattice. JHEP 2015, 2, 186. [arXiv:1412.6411].

5. Brandt, B.B.; Francis, A.; Jaeger, B.; Meyer, H.B. Charge transport and vector meson dissociation across the thermal phase transition in lattice QCD with two light quark flavors. Phys. Rev. 2016, D93, 054510. [arXiv:1512.07249].

6. Ding, H.T.; Kaczmarek, O.; Meyer, F. Thermal dilepton rates and electrical conductivity of the QGP from the lattice. Phys. Rev. 2016, D94, 034504. [arXiv:1604.06712].

7. Greif, M.; Bouras, I., Xu, Z.; Greiner, C. Electric conductivity of the quark-gluon plasma investigated using a perturbative QCD based parton cascade. Phys. Rev. 2014, D90, 094014. [arXiv:1408.7049].

8. Puglisi, A.; Plumari, S.; Greco, V. Electric Conductivity from the solution of the Relativistic Boltzmann Equation. Phys. Rev. 2014, D90, 114009. [arXiv:1408.7043].

9. Arnold, P.; Moore, G.D.; Yaffe, L.G. Transport coefficients in high temperature gauge theories: (I) Leading-log results. JHEP 2000, 11, 001. [hep-ph/0010177].

10. Rougemont, R.; Noronha, J.; Noronha-Hostler, J. Suppression of baryon diffusion and transport in a baryon rich strongly coupled quark-gluon plasma. Phys. Rev. Lett. 2015, 115, 202301. [arXiv:1507.06972].

11. Greif, M.; Greiner, C.; Denicol, G.S. Electric Conductivity of a hot hadron gas from a kinetic approach. Phys. Rev. 2016, D93, 096012. [arXiv:1602.05085].

12. Rougemont, R.; Critelli, R.; Noronha-Hostler, J.; Noronha, J.; Ratti, C. Dynamical vs. equilibrium properties of the QCD phase transition: a holographic perspective. Phys. Rev. 2017, D96, 014032. [arXiv:1704.05558].

13. Monnai, A. Dissipative Hydrodynamic Effects on Baryon Stopping. Phys. Rev. 2012, C86, 014908. [arXiv:1204.4713].

14. Odyniec, G. The RHIC Beam Energy Scan program in STAR and what's next ... J. Phys. Conf. Ser. 2013, $455,012037$. 
15. Adamczyk, L.; Adkins, J.K.; Agakishiev, G.; Aggarwal, M.M.; Ahammed, Z.; Ajitanand, N.N.; Alekseev, I.; Anderson, D.M.; Aoyama, R.; Aparin, A.; et al. (STAR Collaboration). Bulk Properties of the Medium Produced in Relativistic Heavy-Ion Collisions from the Beam Energy Scan Program. Phys. Rev. 2017, C96, 044904. [arXiv:1701.07065].

16. Chapman, S.; Cowling, T.G.; Burnett, D. The Mathematical Theory of Non-Uniform Gases: An Account of the Kinetic Theory of Viscosity, Thermal Conduction and Diffusion in Gases; Cambridge University Press: Cambridge, UK, 1990.

17. Denicol, G.S. Kinetic foundations of relativistic dissipative fluid dynamics. J. Phys. 2014, G41 no. $12,124004$.

18. Greif, M.; Fotakis, J.A.; Denicol, G.S.; Greiner C. Diffusion of conserved charges in relativistic heavy ion collisions. Phys. Rev. Lett. 2018, 120, 242301. [arXiv:1711.08680].

19. Fotakis, J.A.; et al. in preparation.

20. Olive, K.A.; Agashe, K.; Amsler, C.; Antonelli, M.; Arguin, J.-F.; Asner, D.M.; Baer, H.; Band, H.R.; Barnett, R.M.; Basaglia, T.; et al. (Particle Data Group). Review of Particle Physics. Chin. Phys. 2014, C38, 090001.

21. Buss, O.; Gaitanos, T.; Gallmeister, K.; van Hees, H.; Kaskulov, M.; Lalakulich, O.; Larionov, A.B.; Leitner, T.; Weil, J.; Mosel, U. Transport-theoretical Description of Nuclear Reactions. Phys. Rept. 2012, 512, 1-124, [arXiv:1106.1344].

22. Bass, S.A.; Belkacem, M.; Bleicher, M.; Brandstetter, M.; Bravina, L.; Ernst, C.; Gerland, L.; Hofmann, M.; Hofmann, S.; Konopka, J.; et al. Microscopic Models for Ultrarelativistic Heavy Ion Collisions. Prog. Part. Nucl. Phys. 1998, 41, 255-369. [nucl-th/9803035].

23. Bleicher, M.; Zabrodin, E.; Spieles, C.; Bass, S.A.; Ernst, C.; Soff, S.; Bravina, L.; Belkacem, M.; Weber, H.; Stöcker, H.; Greiner, W. Relativistic hadron-hadron collisions in the ultra-relativisticquantum molecular dynamics model. J. Phys. G: Nucl. Part. Phys. 1999, 25, 1859.

24. Xu, Z.; Greiner, C. Shear Viscosity in a Gluon Gas. Phys. Rev. Lett. 2008, 100, 172301. [arXiv:0710.5719].

25. Bouras, I.; Molnar, E.; Niemi, H.; Xu, Z.; El, A.; Fochler, O.; Greiner, C.; Rischke, D.H. Relativistic shock waves in viscous gluon matter. Phys. Rev. Lett. 2009, 103, 032301. [arXiv:0902.1927].

26. Onsager, L. Reciprocal Relations in Irreversible Processes. I. Phys. Rev. 1931, 37, 405-426.

27. Onsager, L. Reciprocal Relations in Irreversible Processes. II. Phys. Rev. 1931, 38, 2265-2279.

(C) 2019 by the authors. Licensee MDPI, Basel, Switzerland. This article is an open access article distributed under the terms and conditions of the Creative Commons Attribution (CC BY) license (http:/ / creativecommons.org/licenses/by/4.0/). 